\title{
Interference Analysis of Metal Conductors for Target-Resonance-Based Detection Method
}

\author{
Lin Chen $(\mathbb{D}$, Huaiqing Zhang $\mathbb{D}$, Yinchun A $\mathbb{D}$, and Wei Song $\mathbb{C}$ \\ State Key Laboratory of Power Transmission Equipment \&System Security and New Technology, Chongqing 400044, China \\ Correspondence should be addressed to Huaiqing Zhang; zhanghuaiqing@cqu.edu.cn
}

Received 1 November 2019; Revised 27 December 2019; Accepted 10 January 2020; Published 19 February 2020

Academic Editor: Yong Xu

Copyright (c) 2020 Lin Chen et al. This is an open access article distributed under the Creative Commons Attribution License, which permits unrestricted use, distribution, and reproduction in any medium, provided the original work is properly cited.

\begin{abstract}
Frequency bifurcation phenomenon is an important input characteristic of the magnetically coupled resonant wireless power transfer (MCR-WPT) system in overcoupling situation. In this paper, frequency bifurcation phenomenon in MCR is extended to the general transmitter-receiver coupling system, and the two main factors affecting the phenomenon are analyzed. Finally, the phenomenon is introduced into the field of signal detection, and a noncontact detection method based on target coil resonance is proposed in this paper. Since the proposed method is a kind of electromagnetic method, the detection system is highly susceptible to the surrounding metal conductors. Therefore, the detection performance of this method under three metal-environment conditions is analyzed, respectively. Simulations and experiments show that the metal barrier can weaken the detection because of the eddy current effect, but as long as the barrier does not completely isolate the two coils, the method still has strong penetrability. Besides, the metal plate placed parallel outside the transmitter has less influence on the detection when compared with the metal barrier, and the metal plate by the side of the transmitter can even enhance the detection effect. As a consequence, this method has strong stability in metal environment.
\end{abstract}

\section{Introduction}

Magnetically coupled resonant (MCR) technology was firstly proposed in 2007 [1], which is a nonradiative, high-efficiency, and medium-distance wireless power transfer (WPT) technology based on magnetic resonance coupling $[1,2]$. It is a great revolution in the field of energy transmission and has been a research hotspot until now. At present, the research of MCR technology mainly aims at improving the energy transmission characteristics of the system, including coil and transmission structure optimization design [3-5] and system control [6].

When the coupling coefficient between the transmitting coil and the receiving coil is too large in the MCR-WPT system, the power transfer will be influenced significantly [7]. In the output side, frequency splitting phenomenon occurs, where the load power-frequency curve changes from a single-peak curve to a double-peak curve, and meanwhile the output power of the energy transfer system cannot reach the maximum [8]. Numerous literatures focus on the causes of frequency splitting [9], the derivation of frequency splitting equations [10], the system structure optimization [11-13], frequency tracking [14], etc., and devote to eliminating frequency splitting or achieving optimal energy transfer in the case of frequency splitting. From the perspective of input characteristic, the compensation capacitors cause the high-order mathematical model of the system to have multiple real roots when the coupling coefficient is greater than a certain value, which is similar to the fold bifurcation of the fast subsystem in some nonlinear systems $[15,16]$. The multiple real roots mentioned above means that the MCR-WPT system has multiple resonant frequencies and this is called frequency bifurcation [10]. Therefore, frequency splitting phenomenon is a description of output characteristics of the system, while the frequency bifurcation phenomenon reflects input characteristics of the system [17]. Both the two phenomena occur in an overcoupling condition, but they are different because the critical coupling coefficients of these two phenomena are not equal. In any case, frequency splitting and bifurcation are undesirable in the power transfer system. 
The previous studies concentrated more on frequency splitting because the output power of the load or the transmission efficiency of the whole system are the research cores from the perspective of energy transfer. But in this paper, we will be more concerned about the frequency bifurcation phenomenon which reflects the input characteristics of the system because we hope to introduce this phenomenon into the application of signal detection, where we cannot directly obtain the characteristics of the secondary side as the detection target is unknown. So, the only access of detecting the target is to analyze the changes of the primary side. Assuming our detection target is equivalent to an RLC circuit or attaches an RLC circuit, the occurrence of frequency bifurcation phenomenon indicates that the RLC target has entered the coupling region of the transmitting coil. Thus, we can achieve the approximate positioning of the unreachable RLC target by this method.

As a detection method based on electromagnetic field theory, this method is easy to be affected by the surrounding metal conductor material in practical applications. Therefore, the metal barrier between the transmitting coil and the detecting target and the metal conductor around the transmitting coil are our main concerns.

The paper is organized as follows: firstly, the contactless detection method based on the target resonance is introduced. Then, the influence of different metal environments on this detection method is analyzed. Finally, the causes of these simulations and experimental phenomena as well as the potential application of the method are discussed.

\section{Frequency Bifurcation}

Figure 1 illustrates a pair of coils $L_{1}$ and $L_{2}$ with internal resistances $R_{1}$ and $R_{2}$. The capacitors $C_{1}$ and $C_{2}$ are added to form a series-series topology. $M$ is the mutual inductance between $L_{1}$ and $L_{2}$.

The impedance of the Primary Side $Z_{1}$ is

$$
Z_{1}=R_{1}+j\left(\omega L_{1}-\frac{1}{\omega C_{1}}\right)=R_{1}+j X_{1} .
$$

And the impedance of the secondary coil is

$$
Z_{2}=R_{2}+j\left(\omega L_{2}-\frac{1}{\omega C_{2}}\right)=R_{2}+j X_{2} .
$$

For the whole system, the input impedance is

$$
Z_{\text {in }}=\left(R_{1}+\frac{\omega^{2} M^{2} R_{2}}{R_{2}^{2}+X_{2}^{2}}\right)+j\left(X_{1}-\frac{\omega^{2} M^{2} X_{2}}{R_{2}^{2}+X_{2}^{2}}\right)=R+j X .
$$

In order to calculate the resonant frequency, the imaginary part of $Z_{\text {in }}$ should be 0 , that is,

$$
\begin{aligned}
\operatorname{Im}\left(Z_{\text {in }}\right)= & \frac{\left(\omega L_{1}-\left(1 / \omega C_{1}\right)\right)\left(R_{2}^{2}+\left(\omega L_{2}-\left(1 / \omega C_{2}\right)\right)^{2}\right)}{R_{2}^{2}+\left(\omega L_{2}-\left(1 / \omega C_{2}\right)\right)^{2}} \\
& -\frac{(\omega M)^{2}\left(\omega L_{2}-\left(1 / \omega C_{2}\right)\right)}{R_{2}^{2}+\left(\omega L_{2}-\left(1 / \omega C_{2}\right)\right)^{2}}=0 .
\end{aligned}
$$

For an MCR system, $\omega_{0}=1 / \sqrt{L_{1} C_{1}}=1 / \sqrt{L_{2} C_{2}}$. In this case, when the two coils are over coupled, the three resonant frequencies due to frequency bifurcation are

$$
\left\{\begin{array}{l}
\omega_{1}=\omega_{0}, \\
\omega_{2,3}=\sqrt{\frac{2 L_{1} L_{2}^{2} \omega_{0}^{2}-R_{2}^{2} L_{1}}{2\left(L_{1} L_{2}^{2}-M^{2} L_{2}\right)} \pm \frac{\sqrt{4 M^{2} L_{1} L_{2}^{3} \omega_{0}^{4}-4 L_{1}^{2} L_{2}^{2} R_{2}^{2} \omega_{0}^{2}+R_{2}^{4} L_{1}^{2}}}{2\left(L_{1} L_{2}^{2}-M^{2} L_{2}\right)}} .
\end{array}\right.
$$

In a more general case, that is, $1 / \sqrt{L_{1} C_{1}} \neq 1 / \sqrt{L_{2} C_{2}}$, and frequency bifurcation will also occur. Assume that a magnetic coupling system has the parameters, as shown in Table 1 . Now we change the compensation capacitor $C_{2}$ to get different resonant frequencies for the secondary coil, which is shown in Table 2.

The impedance phase curve of the transmitting coil is shown in Figure 2(a). The curve has only one zero-crossing point which indicates the resonant frequency of the transmitting coil. When a secondary side coil is added into the system, the phase-frequency curve will change. Figure 2(b) shows the input impedance phase-frequency curves of the magnetic coupling system with different $C_{2}$. It is obvious that the curve has a "drop down" phenomenon and has three zero-crossing points. Comparing Table 2 with Figure 2, we can find that the second zero-crossing frequency in the figure coincides with the resonant frequency $f_{2}$. Hence, this phenomenon can reflect the resonance information of the secondary side. Besides, the analysis shows frequency bifurcation phenomenon also exists in a general transmitterreceiver magnetic coupling system, not only in a MCR system. Figure 2 also demonstrates that with the decrease of $\mathrm{C}_{2}$, the higher resonant frequency leads to a more pronounced bifurcation phenomenon because a small $C_{2}$ can improve the circuit quality factor.

Taking $C_{2}=0.03 \mathrm{nF}$ as an example, the phase-frequency curves with different a mutual inductance $M$ are shown in Figure 3. With the decrease of $M$, frequency bifurcation phenomenon disappears and there is only one resonant frequency for the whole system in around $1.186 \mathrm{MHz}$ which 
TABLE 1: Circuit parameters of the magnetic coupling system.

\begin{tabular}{lcccccc}
\hline Parameter & $R_{1}(\Omega)$ & $L_{1}(\mathrm{nH})$ & $C_{1}(\mathrm{nF})$ & $M(\mathrm{nH})$ & $R_{2}(\Omega)$ & $L_{2}(\mathrm{nH})$ \\
\hline Value & 0.5 & 600 & 30 & 180 & 0.5 & 600 \\
\hline
\end{tabular}

TABLE 2: Resonant frequency $f_{2}$ with different $\mathrm{C}_{2}$.

\begin{tabular}{lcccc}
\hline$C_{2}(\mathrm{nF})$ & 30 & 3 & 0.3 & 0.03 \\
\hline$f_{2}(\mathrm{MHz})$ & 1.1863 & 3.7513 & 11.8627 & 37.5132 \\
\hline
\end{tabular}

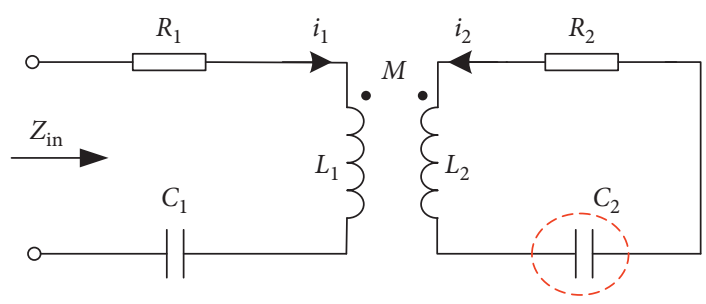

FIGURE 1: A series-series topology of the magnetic coupling system.

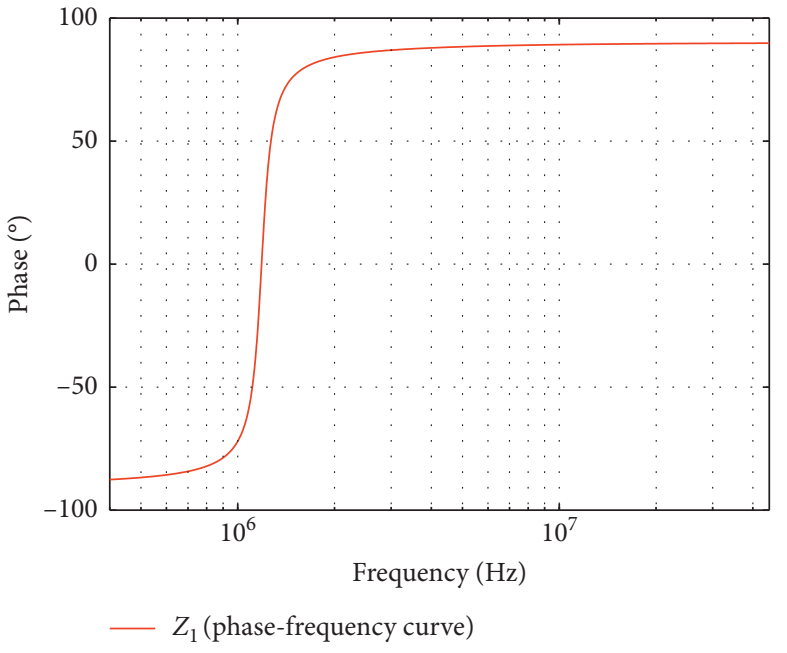

(a)

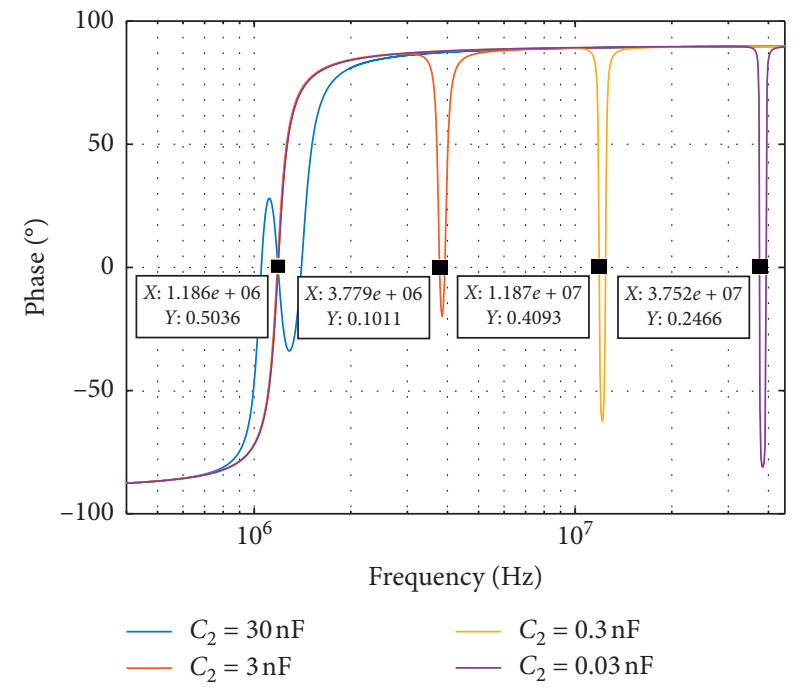

(b)

FIGURE 2: Impedance phase-frequency curve: (a) $Z_{1}$ and (b) $Z_{\text {in }}$ with different $C_{2}$.

is not shown in Figure 3, but there is still a distortion around $f=f_{2}$ in the curve. This distortion also reflects the resonant frequency of the target coil. So, we define this distortion region as the feature region, which indicates the feature of the target coil.

As mentioned above, frequency bifurcation phenomenon and frequency splitting phenomenon are issues that must be avoided in order to realize high-efficiency power transfer. Since this phenomenon reflects the resonant frequency of the secondary coil, from another angle we can apply it in target detection. Supposing a target to be detected is equivalent to an RLC series circuit, when the phase-frequency curve has a distortion, it can be determined that the target enters the coupling region of the transmitting coil, besides, the approximate resonant frequency of the target can be inferred. Obviously, the feature region on the phase-frequency curve of the input impedance is more likely to appear compared with the frequency bifurcation phenomenon. Therefore, using the feature region as the basis for target detection can greatly increase the detection distance, that is, the target body is still likely to be detected under a small coupling coefficient.

As a major parameter affecting the quality factor of the RLC circuit, resistance $R_{2}$ will undoubtedly influence the feature region of the input impedance. Also taking $C_{2}=0.03 \mathrm{nF}$, for example, and $M=180 \mathrm{nH}, R_{2}$ has four values shown in Figure 4. The simulation results in Figure 4 demonstrate that a high-quality factor RLC target is much easier to be detected. 


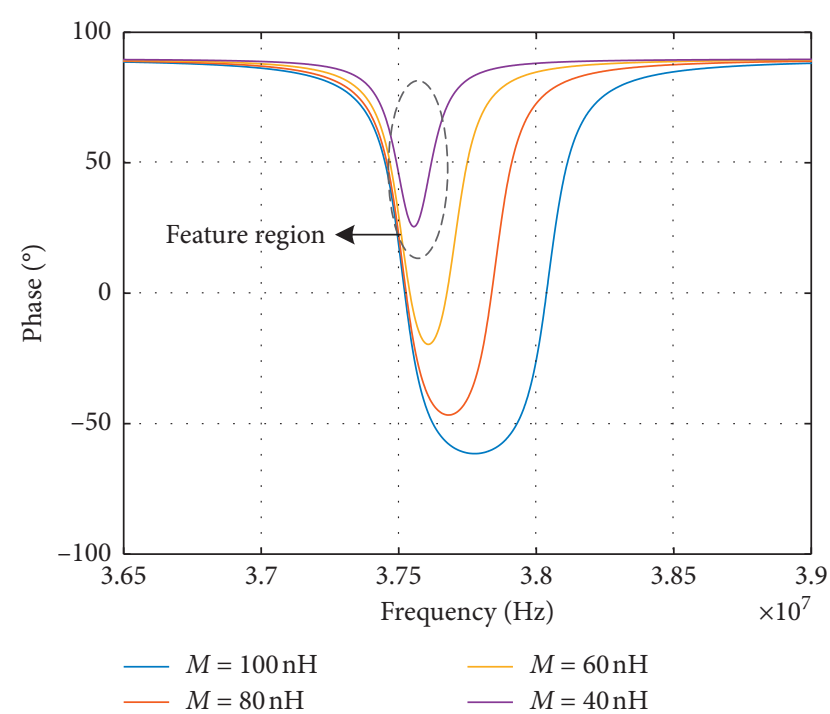

Figure 3: The phase-frequency curves with different $M$ when $C_{2}=0.03 \mathrm{nF}$.

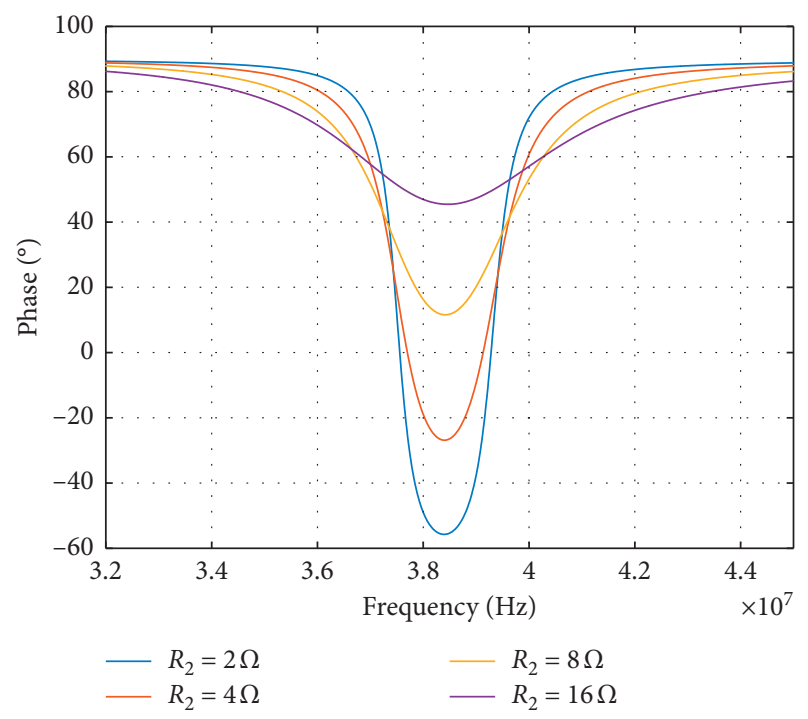

FIgURE 4: The phase-frequency curves with different $R_{2}$ when $C_{2}=0.03 \mathrm{nF}$.

\section{Interference Analysis of Metal Environment}

As an electromagnetic-based detection method, the method is highly sensitive to the detection environment, especially the influence of surrounding metal conductors. Therefore, this paper explores the detection performance in various metal environments. The system has two copper coils, transmitting coil and target coil. Other parameters of the system are shown in Table 3.

We chose a 304 stainless steel plate with the size of $300 \mathrm{~mm} * 300 \mathrm{~mm} * 0.3 \mathrm{~mm}$ and place the metal plate in different positions, as shown in Figure 5. The metal plate in Figure 5(a) is placed in parallel between the two coils. In Figure 5(b), the metal plate is placed parallel outside of the transmitting coil. And the metal plate is placed by the side of the transmitting coil is shown in Figure 5(c).
TABLE 3: Structural parameters of transmitting coil and target coil.

\begin{tabular}{lcccc}
\hline Parameter & Turn & $\begin{array}{c}\text { Coil radius } \\
(\mathrm{mm})\end{array}$ & $\begin{array}{c}\text { Wire radius } \\
(\mathrm{mm})\end{array}$ & $\begin{array}{c}\text { Coils distance } \\
(\mathrm{mm})\end{array}$ \\
\hline Value & 1 & 102.5 & 1.25 & 200 \\
\hline
\end{tabular}

3.1. Metal Barrier. This section firstly analyzes the influence of the metal barrier on values of $L_{2}, M$, and $R_{2}$ in 5 cases, as shown in Figure 6.

MAXWELL simulation results are shown in Figure 7. Abscissa $d$ varying from $1 \mathrm{~cm}$ to $19 \mathrm{~cm}$ is the distance between the barrier and the transmitter. Without the barrier, $M=15.314 \mathrm{nH}, R_{2}=9.4443 \mathrm{~m} \Omega$, and $L_{2}=598.56 \mathrm{nH}$. Simulation results demonstrate that the metal barrier can reduce $M$, and the more severely the two coils are shielded by the metal barrier, the smaller the mutual inductance is. For $R_{2}$, although it is difficult to find an exact law from the simulation results because the curves are messy, we can see that $R_{2}$ increases due to the metal barrier. Therefore, when the metal plate functions as a barrier, the detection performance of the system will be weakened. The effect of the metal barrier on $L_{2}$ will change the resonance frequency of the secondary target, thereby changing the position of the feature region. Figure 7 illustrates the closer the barrier is to the secondary side, the smaller $L_{2}$ is and the faster it decreases.

Taking the case of $75 \%$ for instance, the influence of the metal plate on the phase-frequency curve of the system is investigated. Figure 8 shows the detection system, where the device is the R\&S ZND vector net analyzer. $C_{1}=30 \mathrm{nF}$ and $C_{2}=30 \mathrm{pF}$. Without the metal barrier, the lowest point of the feature region is $\left(37.74 \mathrm{MHz}, 57.3^{\circ}\right)$. That is, the phase curve drops from around $90^{\circ}$ to $57.3^{\circ}$.

Figure 9 demonstrates the variation of the input impedance phase-frequency curve when the metal barrier is at different locations. It is obvious that the metal barrier weakens the performance of the system. The lowest point of the feature regions changes from $57.3^{\circ}$ to about $84^{\circ}$. Closer distance between the metal barrier and either of the two coils will have greater impact on the detection. In addition, the resonance frequency of the target coil changes because of the constant compensation capacitor $C_{2}$ and the varying $L_{2}$, so the feature region is frequency-shifted. The closer the barrier is to the target coil, the severer the frequency shift is. All the experiment results are consistent with the previous MAXWELL simulation results.

In order to investigate the penetrability of the detection method in the presence of the metal barrier, the barrier is placed $2 \mathrm{~cm}$ away from the transmitting coil. Here, a rule is made that when the phase angle changes by about $5^{\circ}$, the feature region is considered to be observable. The maxima detection distance without the metal barrier is $30 \mathrm{~cm}$ according to the experiment. In this section, except the former metal plate, we use another three different barriers to observe the maximum detection distance. The three different barriers are metal grid with different grid sizes of $25 \mathrm{~mm} * 25 \mathrm{~mm}, 50 \mathrm{~mm} * 50 \mathrm{~mm}$, and $100 \mathrm{~mm} * 100 \mathrm{~mm}$, respectively. The metal conductor diameter of the metal grid is $2 \mathrm{~mm}$. The experimental results are shown in Table 4 . 


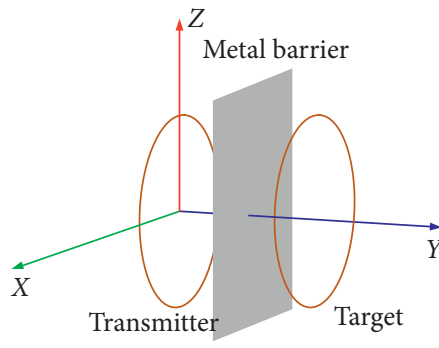

(a)

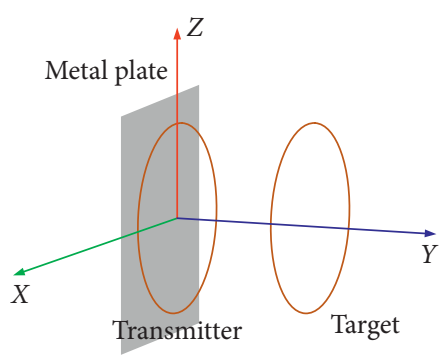

(b)

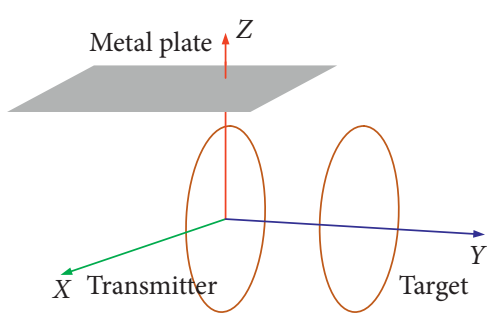

(c)

Figure 5: The metal plate is in different places. (a) Barrier situation; (b) metal plate is parallel outside the transmitter; and (c) metal plate is by the side of the transmitter.

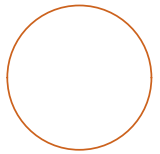

(a)

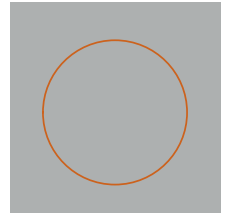

(b)

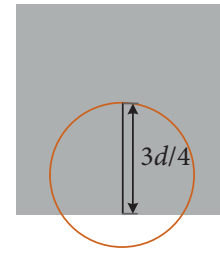

(c)

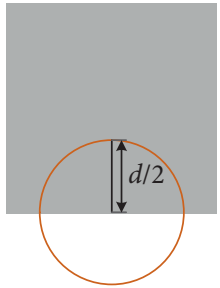

(d)

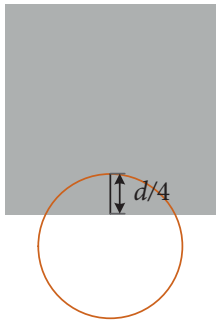

(e)

Figure 6: (a) Without barrier; (b) 100\% shielded; (c) 75\% shielded; (d) 50\% shielded; (e) 25\% shielded.

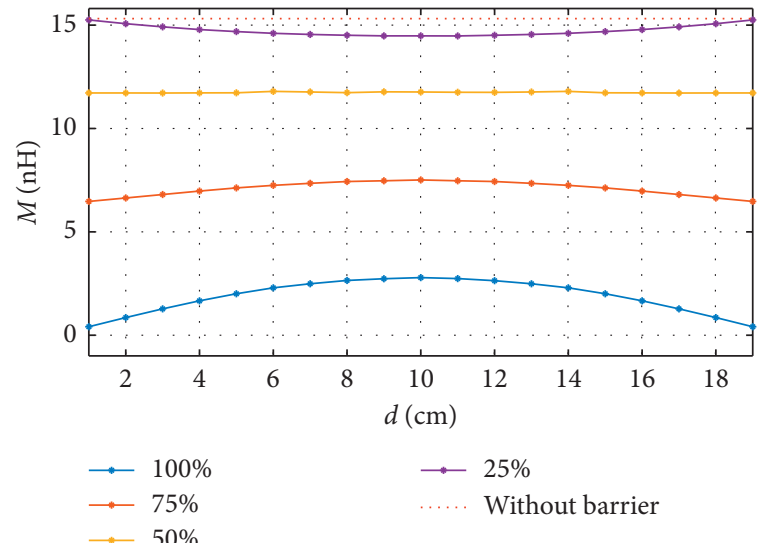

(a)

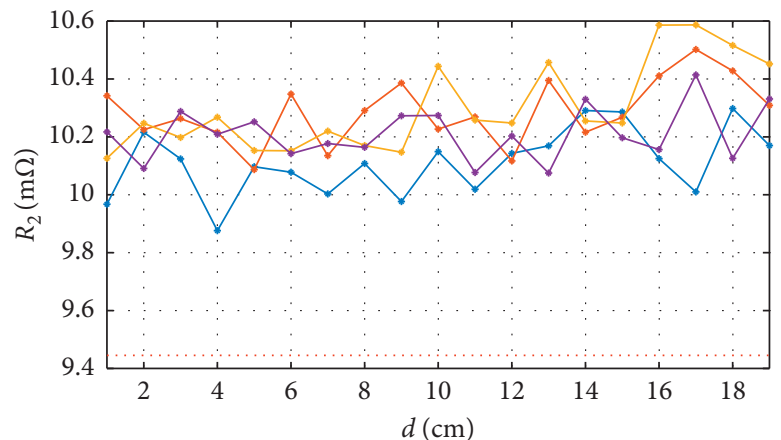

$\longrightarrow \quad 100 \%$
$\rightarrow-75 \%$
$\longrightarrow \quad 50 \%$

$\rightarrow 25 \%$

Without barrier

(b)

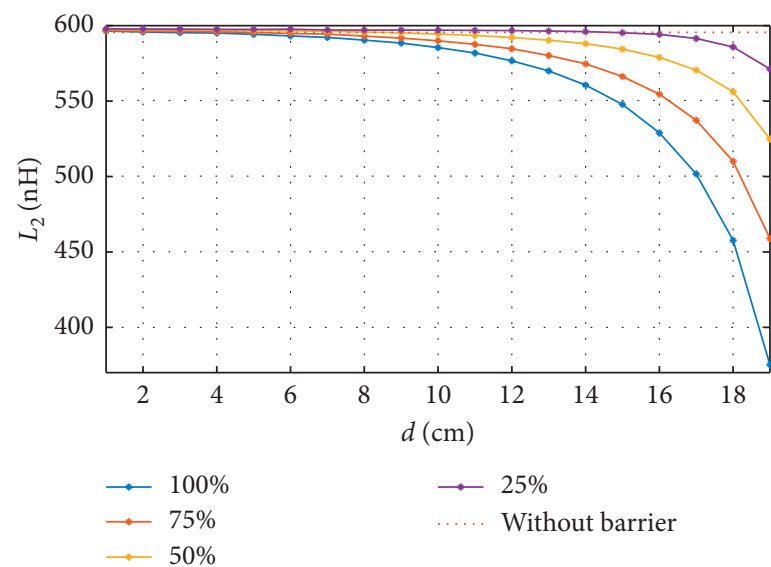

(c)

Figure 7: Parameters curves with the influence of the metal barrier. 


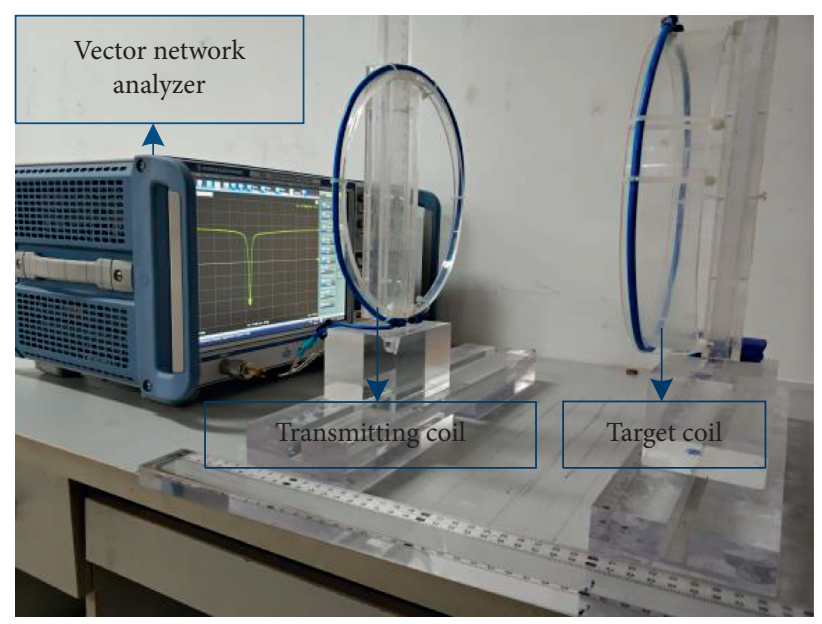

FIGURE 8: Experimental setup of the detection system.

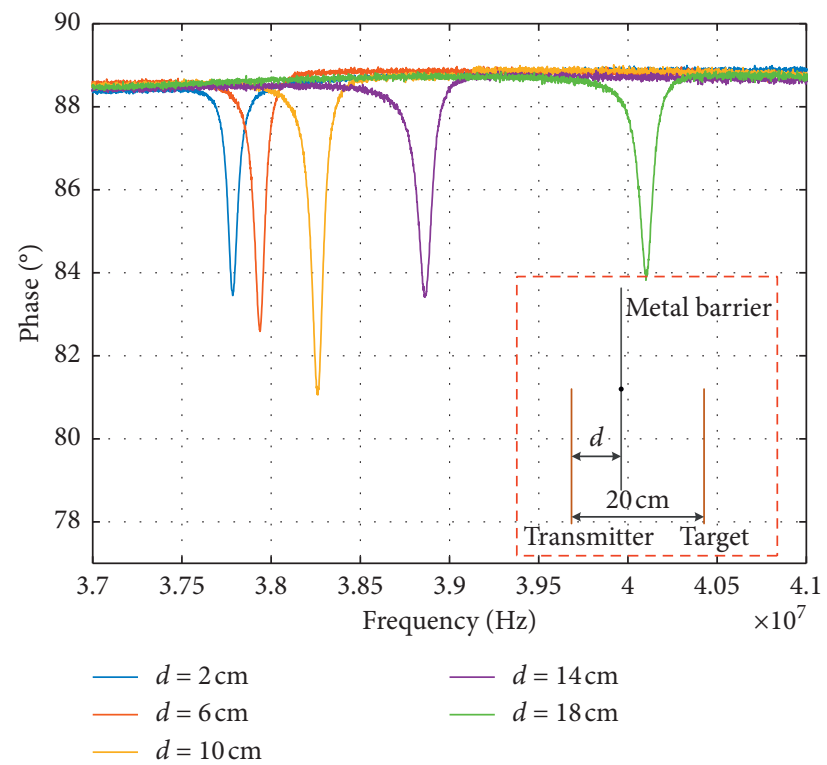

FIGURE 9: Experiment results of the input impedance phase-frequency curve with different $d$.

The method has a maximum detection distance of $30 \mathrm{~cm}$ without the barrier, which is about 3 times of the detection coil radius. When the barrier is $2 \mathrm{~cm}$ away from the transmitter, the maximum detection distance will decrease. It is the worst case when the two coils are completely obscured by a metal plate. But this situation does not exist in practical applications, and we will not discuss it.

For all the four kinds of barriers, when blocking 25\% of the radius, it has little influence on the detection performance. When blocking $75 \%$ of the radius, the detection distance is at least $2 / 3$ of that without barrier. For metal grid barriers, the denser the mesh is, the worse the detection effect is. Fortunately, when the $25 * 25$ mesh is $100 \%$ shielding the coil, it can achieve the detection distance of $13.5 \mathrm{~cm}$, which is greater than the radius of the coil. In a word, it can still achieve a relatively long-distance detection as long as the barrier does not completely block the coils.
3.2. Metal Plate is Parallel outside the Transmitter. The previous simulation reveals that the closer the metal plate is to the system, the greater the impact on the system parameters is. When the metal plate is located outside the transmitting coil in parallel, it is relatively far away from the secondary side, so it has little influence on the parameters of the target coil. But because of the eddy current effect, its impact on mutual inductance $M$ is not negligible. Table 5 shows the simulation values of $M$ when the metal plate is at different locations. Since $M=15.314 \mathrm{nH}$ without the metal plate, it can be found from Table 5 that if the metal plate is close to the transmitter, it has great impact on $M$.

Figure 10 demonstrates that when the metal plate is $2 \mathrm{~cm}$ from the transmitter, the phase of the lowest point in the feature region changes from $57.3^{\circ}$ to $71.15^{\circ}$. When $d=8 \mathrm{~cm}$, the metal plate has little influence on the detection. 
TAвLE 4: The maximum detection distances when different barriers are placed $2 \mathrm{~cm}$ away from the transmitter.

\begin{tabular}{lcccc}
\hline & Metal plate & \multicolumn{2}{c}{ Metal grid } \\
& & $25 \mathrm{~mm} * 25 \mathrm{~mm}$ & $50 \mathrm{~mm} * 50 \mathrm{~mm}$ & $100 \mathrm{~mm} * 100 \mathrm{~mm}$ \\
\hline $100 \%$ shielded & - & $13.5 \mathrm{~cm}$ & $29.5 \mathrm{~cm}$ & $23 \mathrm{~cm}$ \\
$75 \%$ shielded & $20.0 \mathrm{~cm}$ & $21.5 \mathrm{~cm}$ & $23.5 \mathrm{~cm}$ & $25.5 \mathrm{~cm}$ \\
$50 \%$ shielded & $25.5 \mathrm{~cm}$ & $26.5 \mathrm{~cm}$ & $27.5 \mathrm{~cm}$ & $28 \mathrm{~cm}$ \\
$25 \%$ shielded & $28.0 \mathrm{~cm}$ & $29.0 \mathrm{~cm}$ & $29.5 \mathrm{~cm}$ & $29.5 \mathrm{~cm}$ \\
\hline
\end{tabular}

TABle 5: $M$ varies with the different distances between the transmitter and the metal plate.

\begin{tabular}{lcccc}
\hline$d(\mathrm{~cm})$ & 2 & 4 & 6 & 8 \\
\hline$M(\mathrm{nH})$ & 5.8023 & 9.3263 & 11.481 & 12.811 \\
\hline
\end{tabular}

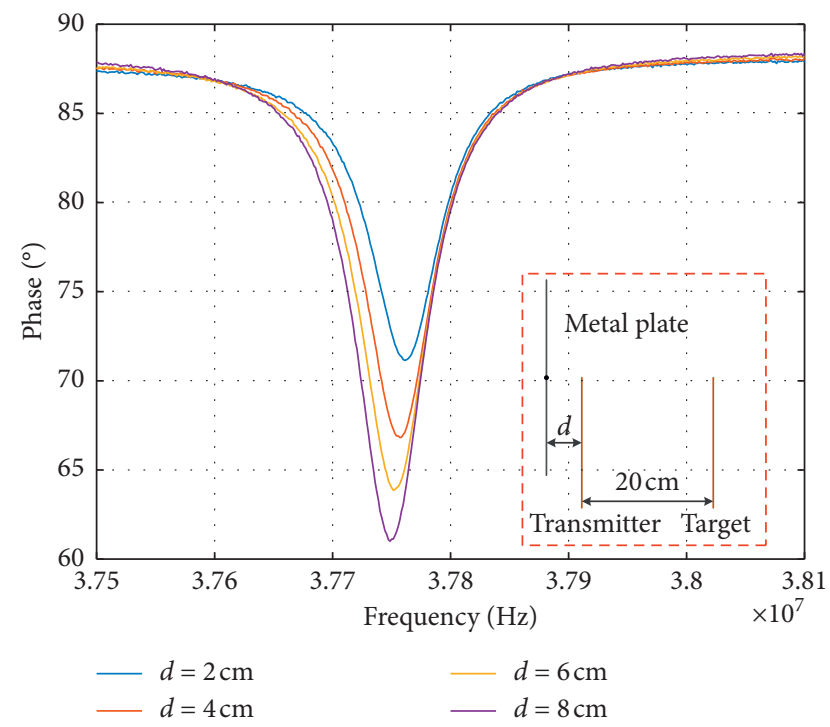

FIGURE 10: Influence of the metal plate which is parallel outside the transmitter on the feature region.

3.3. Metal Plate is by the Side of the Transmitter. Table 6 shows the effect of the metal plate on the mutual inductance $M$ in the condition shown in Figure 5(c). When $d=20 \mathrm{~cm}$, the whole metal plate is located on the left side of the transmitting coil, and $M$ is slightly reduced. When the metal plate moves to the right, $M$ will decrease and then increase. Figure 11 illustrates the experiment results. As $d$ decreases, the detection effect is slightly weakened firstly and then greatly enhanced. When $d=0 \mathrm{~cm}$, the lowest point of the feature region is reduced from $57.3^{\circ}$ to $48.24^{\circ}$. In this case, the presence of the metal plate is advantageous for detection.

\section{Discussion}

The effect of the metal conductor on the detection system is actually the effect of the magnetic field generated by the eddy current in the metal conductor on the system parameters. In order to clearly observe the eddy current induced when the metal conductor is at different positions, a current with the
TABLE 6: $M$ varies with the change of the plate position.

\begin{tabular}{lccccc}
\hline$d(\mathrm{~cm})$ & 20 & 15 & 10 & 5 & 0 \\
\hline$M(\mathrm{nH})$ & 15.125 & 14.589 & 15.229 & 15.794 & 17.396 \\
\hline
\end{tabular}

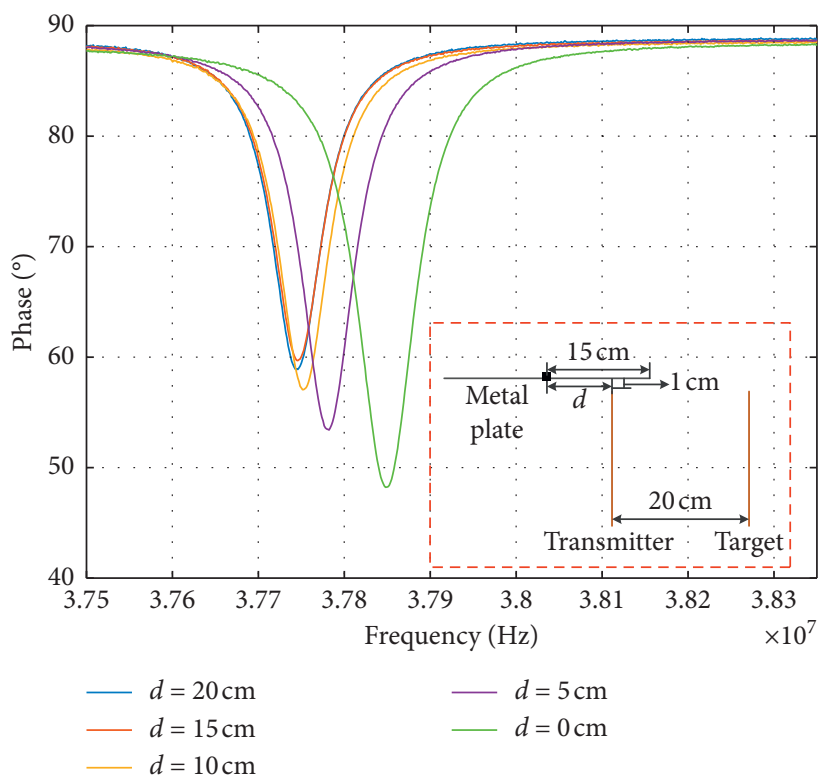

FIGURE 11: Influence of the metal plate on the feature region when it is by the side of the transmitter vertically.

amplitude of $10 \mathrm{~A}$ is applied to the transmitting coil at a frequency of $10 \mathrm{MHz}$. The eddy current in the metal plate is shown in Figure 12. When the metal plate is placed parallel to the transmitting coil, a current in the opposite direction to the original current is induced, and a reverse magnetic field is generated, which reduces the magnetic flux between the two coils, so that the detection effect is weakened. When the metal plate is perpendicular to the transmitting coil, as shown in Figure 12(c), two different directions of current are induced in the metal plate. The part of the metal plate located on the left side of the transmitting coil induces a clockwise current, and the right-side part induces a counterclockwise current which is greater than the left clockwise current. In this occasion, the magnetic field generated by the right counterclockwise current is a forward magnetic field, which increases the magnetic flux between the two coils, so the detection effect is enhanced.

The detection method in this paper is not proposed blindly. So far, there is no mature and effective method for 


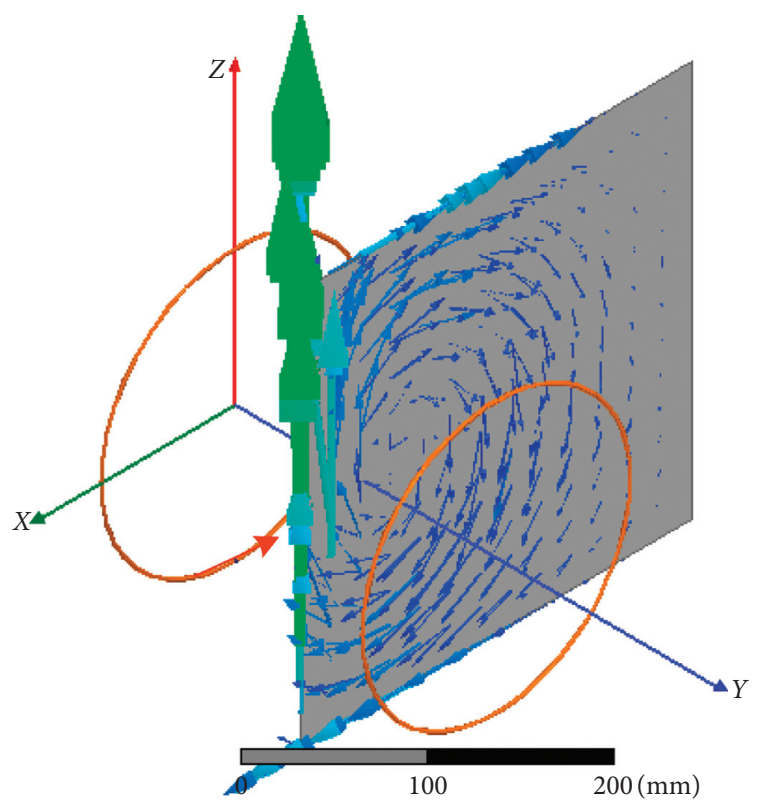

(a)

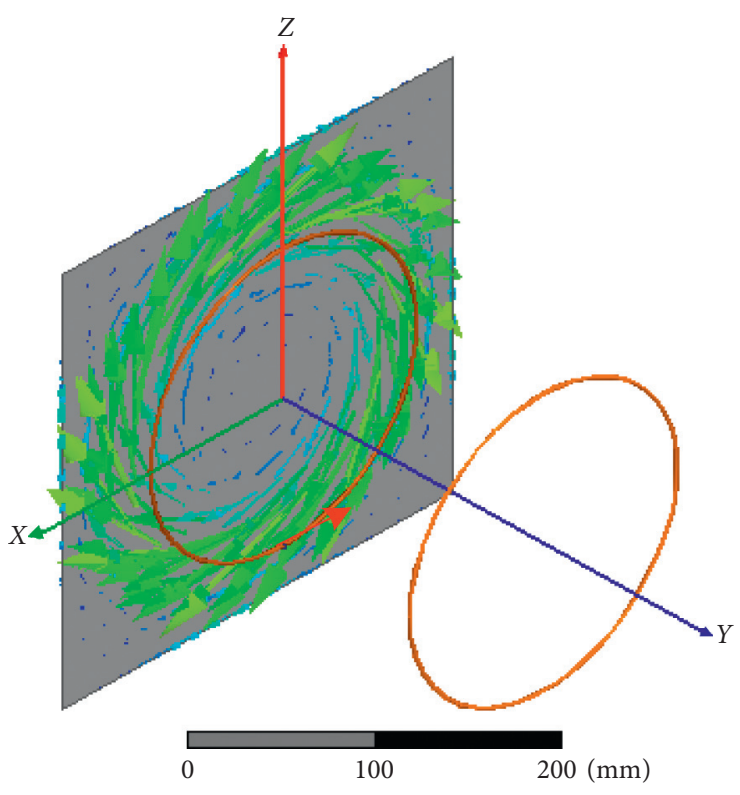

(b)

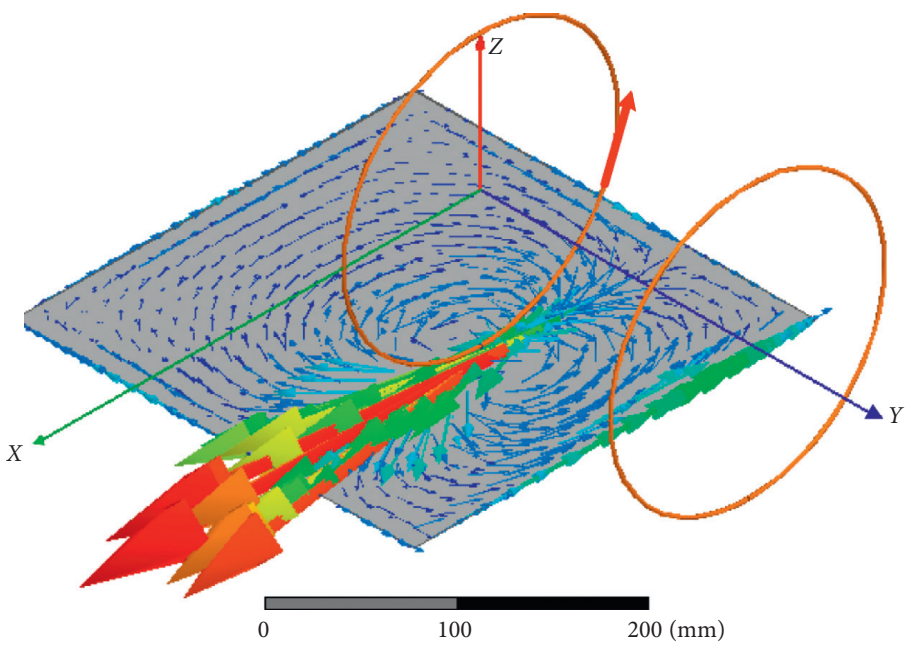

J [A_per_m2]

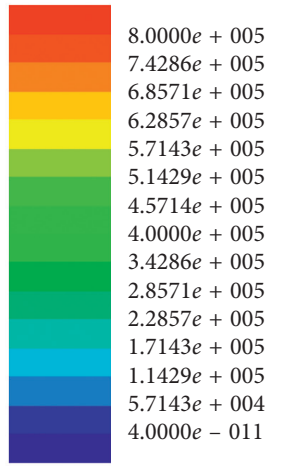

(c)

FIGURE 12: Eddy current in the metal plate under different conditions. (a) Barrier situation; (b) metal plate is parallel outside the transmitter; (c) metal plate is by the side of the transmitter.

the nonexcavation breakpoint diagnosis of the grounding grid. The most widely studied approaches include the method based on theory of electric network, method based on the electromagnetic field theory, and transient electromagnetic method (TEM). The first approach often fails in practical because the changes of measured parameters are poorly observable. And the method based on electromagnetic theory requires that the grounding grid has enough leads to inject current. The TEM method is sensitive to surrounding metal equipment, and the reinforced concrete layer makes it difficult to couple the transmitted signal to the grounding grid conductor [18].
Figure 13 shows the grounding gird in a substation. When the grounding grid is intact, it can be equivalent to an RL coil. If there is a breakpoint, the mesh where the breakpoint is located can be equivalent to an RLC circuit, which is consistent with the circuit model of this paper. The metal equipment on the ground of the substation is equivalent to the metal conductors outside the transmitting coil, while the reinforced concrete can be regarded as the metal barrier. Therefore, the research in this paper is the basis of the new method of grounding network breakpoint diagnosis. The stability of this method in the metal environment also provides the possibility for its practical application. 


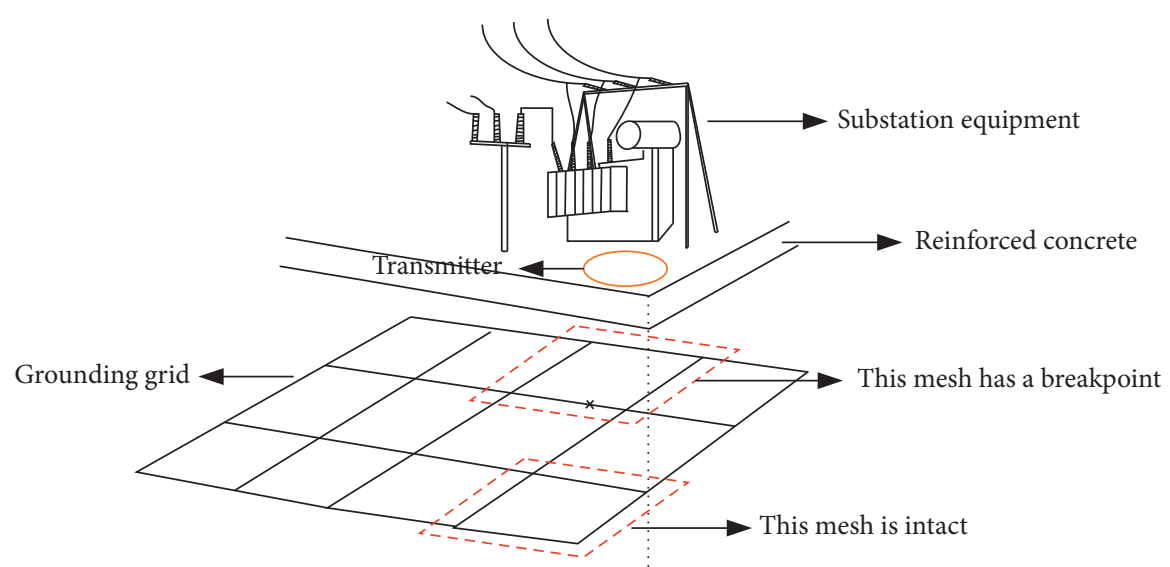

FIgURE 13: Schematic diagram of grounding grid breakpoint detection in a substation.

\section{Conclusions}

The following conclusions are drawn from the analysis of this paper.

(1) Frequency bifurcation phenomenon exists not only in a MCR system, but also in a general transmitterreceiver coupling system, where the transmitting coil and the secondary coil have different self-resonant frequencies. The first zero-crossing point of the frequency bifurcation is determined by the resonant frequency of the transmitting coil, while the second and the third zero-crossing points are determined by the secondary side, so this phenomenon reflects the resonant information of the secondary coil. Besides, the increase of mutual inductance $M$, and the decrease of the secondary side internal resistance $R_{2}$ and compensation capacitor $C_{2}$ can enhance this phenomenon.

(2) The two zero-crossing points near the secondary side resonance frequency will vanish and the frequency bifurcation phenomenon also will disappear with the decrease of $M$. However, there is still a distortion region defined as the feature region near the selfresonant frequency $f_{2}$ of the secondary coil. Therefore, this paper proposes a detection method based on the target resonance.

(3) A metal plate parallel to the transmitting coil has a great influence on the detection, especially when it is close to the transmitting coil. However, as long as the transmitting coil is not completely blocked by the metal plate, the detection distance is still considerable. The metal plate which is by the side of the transmitting coil has little effect on the detection performance or even enhances it. In short, the method has a strong penetrating ability for metal barriers and a strong stability in a metal environment.

\section{Data Availability}

The data used to support the findings of this study are included within the article.

\section{Conflicts of Interest}

The authors declare that there are no conflicts of interest regarding the publication of this paper.

\section{Acknowledgments}

This work was partly supported by the National Natural Science Foundation of China (Nos. 51577016, 51377174, and 51877014).

\section{References}

[1] A. Kurs, A. Karalis, R. Moffatt, J. D. Joannopoulos, P. Fisher, and M. Soljacic, "Wireless power transfer via strongly coupled magnetic resonances," Science, vol. 317, no. 5834, pp. 83-86, 2007.

[2] R. E. Hamam, A. Karalis, J. D. Joannopoulos, and M. Soljačić, "Efficient weakly-radiative wireless energy transfer: an EITlike approach," Annals of Physics, vol. 324, no. 8, pp. 17831795, 2009.

[3] J. Kim, H. Son, K. Kim, and Y. Park, "Efficiency analysis of magnetic resonance wireless power transfer with intermediate resonant coil," IEEE Antennas and Wireless Propagation Letters, vol. 10, pp. 389-392, 2011.

[4] B. H. Waters, B. J. Mahoney, V. Ranganathan, and J. R. Smith, "Power delivery and leakage field control using an adaptive phased array wireless power system," IEEE Transactions on Power Electronics, vol. 30, no. 11, pp. 6298-6309, 2015.

[5] D. Lin, C. Zhang, and S. Y. R. Hui, "Mathematic analysis of omnidirectional wireless power transfer-part-II three-dimensional systems," IEEE Transactions on Power Electronics, vol. 32, no. 1, pp. 613-624, 2017.

[6] N. Ha-Van and C. Seo, "Analytical and experimental investigations of omnidirectional wireless power transfer using a cubic transmitter," IEEE Transactions on Industrial Electronics, vol. 65, no. 2, pp. 1358-1366, 2018.

[7] R. Huang, B. Zhang, D. Qiu, and Y. Zhang, "Frequency splitting phenomena of magnetic resonant coupling wireless power transfer," IEEE Transactions on Magnetics, vol. 50, no. 11, pp. 1-4, 2014.

[8] J. Wang, M. Leach, E. G. Lim, Z. Wang, and Y. Huang, "Investigation of magnetic resonance coupling circuit topologies for wireless power transmission," Microwave and Optical Technology Letters, vol. 61, no. 7, pp. 1755-1763, 2019. 
[9] A. P. Sample, D. A. Meyer, and J. R. Smith, "Analysis, experimental results, and range adaptation of magnetically coupled resonators for wireless power transfer," IEEE Transactions on Industrial Electronics, vol. 58, no. 2, pp. 544-554, 2011.

[10] W.-Q. Niu, J.-X. Chu, W. Gu, and A.-D. Shen, "Exact analysis of frequency splitting phenomena of contactless power transfer systems," IEEE Transactions on Circuits and Systems I: Regular Papers, vol. 60, no. 6, pp. 1670-1677, 2013.

[11] S. Huang, Z. Li, and K. Lu, "Frequency splitting suppression method for four-coil wireless power transfer system," IET Power Electronics, vol. 9, no. 15, pp. 2859-2864, 2016.

[12] X. Y. Zhang, C.-D. Xue, and J.-K. Lin, "Distance-insensitive wireless power transfer using mixed electric and magnetic coupling for frequency splitting suppression," IEEE Transactions on Microwave Theory and Techniques, vol. 65, no. 11, pp. 4307-4316, 2017.

[13] Y.-L. Lyu, F.-Y. Meng, G.-H. Yang et al., "A method of using nonidentical resonant coils for frequency splitting elimination in wireless power transfer," IEEE Transactions on Power Electronics, vol. 30, no. 11, pp. 6097-6107, 2015.

[14] H. Li, J. Li, K. Wang, W. Chen, and X. Yang, "A maximum efficiency point tracking control scheme for wireless power transfer systems using magnetic resonant coupling," IEEE Transactions on Power Electronics, vol. 30, no. 7, pp. 39984008, 2015.

[15] X. Han, Y. Liu, Q. Bi, and J. Kurths, "Frequency-truncation fast-slow analysis for parametrically and externally excited systems with two slow incommensurate excitation frequencies," Communications in Nonlinear Science and Numerical Simulation, vol. 72, pp. 16-25, 2019.

[16] X. Han, Q. Bi, P. Ji, and J. Kurths, "Fast-slow analysis for parametrically and externally excited systems with two slow rationally related excitation frequencies," Physical Review E, vol. 92, no. 1-1, p. 012911, 2015.

[17] M. Iordache, L. Mandache, D. Niculae, and L. Iordache, "On exact circuit analysis of frequency splitting and bifurcation phenomena in wireless power transfer systems," in Proceedings of the 2015 International Symposium on Signals, Circuits and Systems (ISSCS), pp. 1-4, Iasi, Romania, July 2015.

[18] C. Yu, Z. Fu, X. Hou, H.-M. Tai, and X. Su, "Break-point diagnosis of grounding grids using transient electromagnetic apparent resistivity imaging," IEEE Transactions on Power Delivery, vol. 30, no. 6, pp. 2485-2491, 2015. 